\title{
Correction to: Osteogenic prospective of deriving human dental stem cells in collagen matrix boost
}

\author{
Tong-Jing Fang ${ }^{1}$ - Ding-Han Wang ${ }^{2,3} \cdot$ Chia-Yu Wang $^{4} \cdot$ Raju Poongodi $^{3}$ - Nien-Hsien Liou ${ }^{5}$ Jiang-Chuan Liu ${ }^{5}$. \\ Ming-Lun Hsu ${ }^{2} \cdot$ Po-Da Hong ${ }^{1} \cdot$ Shih-Fang Yang ${ }^{2} \cdot$ Meng-Lun Liu ${ }^{6}$
}

Published online: 26 June 2018

(c) Springer Science+Business Media, LLC, part of Springer Nature 2018

Correction to: Journal of Materials Science: Materials in Medicine (2017) 28:192

https://doi.org/10.1007/s10856-017-6001-9

The original version of this article unfortunately contained a mistake. The country was incorrect in the authors affiliations. It should read as "ROC". The corrected affiliations are given below.

The original article can be found online at https://doi.org/10.1007/ s10856-017-6001-9.

Shih-Fang Yang

sfyang2017@gmail.com

$\triangle$ Meng-Lun Liu

doc20054@gmail.com

1 Graduate Institute of Applied Science and Technology, National Taiwan University of Science and Technology, Taipei, Taiwan, ROC

2 School of Dentistry, National Yang-Ming University, Taipei, Taiwan, ROC

3 Lab of Adult Stem Cell and Tissue Regeneration National Defense Medical Center, Taipei, Taiwan, ROC

4 Department of Materials Science and Engineering, National Taiwan University of Science and Technology, Taipei, Taiwan, ROC

5 Department of Biology and Anatomy, National Defense Medical Center, Taipei, Taiwan, ROC

6 Department of Surgery, Cheng Hsin General Hospital, Taipei, Taiwan, ROC 